\title{
Erratum: Critical Current in a System of Two Superconductors Connected by a Short Small-Diameter Normal Metal Bridge (Pis'ma Zh. Éksp. Teor. Fiz. 76, 380 (2002) [JETP Lett. 76, 321 (2002)]
}

\author{
Yu. N. Ovchinnikov and A. I. Larkin
}

PACS numbers: 74.50.+r; 74.25.Sv

In our article published in vol. 76, no. 5, pp 321325 , we studied current states in the SNS junctions. The solutions described by Eq. (8) in the low-frequency limit do not agree with the high-frequency results known from perturbation theory. We have concluded that the states described by our solution do not exist and that the only correct solution is that found by I. Kulik and A. Omel'yanchuk.

We are grateful to M. Fer̆gel'man and Ya. Fominov for pinpointing this fact.

Yu. Ovchinnikov and A. Larkin 